\title{
A New Approach for Motion Correction in SPECT Imaging
}

\author{
Hanno Schumacher and Bernd Fischer \\ University of Lübeck, Institute of Mathematics, \\ Wallstraße 40, 23560 Lübeck, Germany \\ Email: schumaha@math.uni-luebeck.de
}

\begin{abstract}
Due to the long imaging times in SPECT, patient motion is inevitable and constitutes a serious problem for any reconstruction algorithm. The measured inconsistent projection data lead to reconstruction artifacts which can significantly affect the diagnostic accuracy of SPECT if not corrected. Among the most promising attempts for addressing this cause of artifacts is the so-called data-driven motion correction methodology. But even this algorithm is restricted to the correction of abrupt rigid patient motion and exclusive correction of gradual motion, which may lead to unsatisfactory results. In this note we present for the first time a motion correction approach which overcomes the mentioned restrictions. The new approach is based on the super-resolution methodology. To demonstrate the performance of the proposed scheme, corrections of abrupt and gradual motion are presented.
\end{abstract}

\section{Introduction}

In Single Photon Emission Computed Tomography (SPECT), the imaging time is typically in the range of 5-30 minutes. Here, patient movement, which has frequently been reported in clinical applications [1], constitutes a serious problem for any reconstruction scheme. The movements cause misalignment of the projection frames, which degrades the reconstructed image and may introduce artefacts. These motion artefacts may significantly affect the diagnostic accuracy $[2,3,4]$. Different methods have been proposed for the correction of motion in SPECT studies. These methods may be divided into three categories. The first two approaches do produce motion corrected projections and thus may be used in conjunction with any reconstruction method. The first approach is purely hardware based, like, for example the triple scan [5] or dual scan [6] protocol. The second approach corrects for the patient motion by using a computational method applied within the projection-space $[7,8]$. It should be noted, that due to the projection geometry the latter method is not able to compensate for rotational movement. In this paper, we are concerned with the third methodology. Here the correction is performed in the image space. A widely used member out of this class is the so-called data driven motion correction (DDMC) approach $[9,10]$. It can handle full rigid-body motion. To start the scheme, it is assumed that the point in time of the rigid-body motion of the patient during the SPECT 
imaging is known. Once the point in time of the motion is known it needs to be corrected. The idea is to subdivide the projection data into subsets or motionsets where no motion has been detected and to estimate the motion in between these subsets accordingly by using a partial reconstruction of the subset containing the largest number of projections and calculate a suitable transformation in order to fit it to the other subsets. To this end the rigid-body parameters after the $i$ th movement of the patient are stored in the vector $T_{i}$. Furthermore, all projections that were measured between the $i$ th and the $i+1$ st movement are collected in the projection set $\mathbf{P}_{i}$. The image, which has been in the course of the algorithm reconstructed up to the $i$ th step, is denoted by $\mathbf{f}^{(i)}$. This image has to be corrected with respect to the next object position $T_{i+1}$. The result is denoted by $\mathbf{f}^{(i)}\left(T_{i+1}\right)$. Next the partial reconstruction $\mathbf{f}^{(i)}\left(T_{i+1}\right)$ is updated with the help of measured projections $\mathbf{P}_{i+1}$ via

$$
\mathbf{f}^{(i+1)}=\mathbf{R}\left[\mathbf{P}_{i+1}, \mathbf{f}^{(i)}\left(T_{i+1}\right)\right]
$$

where $\mathbf{R}$ denotes a reconstruction algorithm. Ideally, the resulting image $\mathbf{f}^{(m)}$ should contain less motion artefacts. But this approach has two main disadvantages. The first is the needed information about the point in time of the patient motion during the SPECT imaging, and the second is the motion estimation using partial reconstructions. Due to this the quality of the motion estimation depends on the quality of the partial reconstruction, which is only of good quality if $\frac{1}{3}$ of all projections are in one subset. To overcome these disadvantages we present a new approach for motion correction in SPECT imaging that combines reconstruction and motion correction.

\section{State of the art and new contribution}

The data-driven approach, as outlined in the introduction, constitutes the state of the art in motion correction approaches. Nevertheless, it does produce nonsatisfactory results if the time of movement is not correctly estimated and if there are too many movements such that the set projections without movement does not contain enough information to produce a meaningful partial reconstruction. Here, we present a motion correction approach which does combine reconstruction and motion correction in using the super-resolution methodology to advantage. To our best knowledge, this has not been conducted before. Moreover, the novel approach does overcome the just mentioned shortcomings of schemes working solely on the raw data.

\section{Methods}

Let us now describe the main idea of combining reconstruction and motion correction within SPECT imaging. Given SPECT raw data $\mathbf{g}$, we are searching for a reconstructed image $\mathbf{f}$ and for possible motion parameters $\gamma$. The whole problem 
may be formulated as an optimization problem

$$
J\left(\begin{array}{l}
\mathbf{f} \\
\gamma
\end{array}\right)=\frac{1}{2} \sum_{i=1}^{K}\left\|\mathbf{A}_{i} \mathbf{f}\left(\gamma_{i}\right)-\mathbf{g}_{i}\right\|_{2}^{2}+R(\mathbf{f}) \rightarrow \min \quad \text { s.t. } \mathbf{f} \geq 0
$$

Some comments are in order. Here, $\mathrm{K}$ denotes the number of motion-sets. As it will be shown in the result section, the actual value of $K$ is not critical and may be overestimated. Note, that $K$ just denotes the number and not the precise time of motion. Furthermore, $\mathbf{A}_{i}$ denotes the $i$-th part of projection operator $\mathbf{A}$, simulating a SPECT imaging. At least $\gamma_{i}$ denotes the motion parameters and $\mathbf{g}_{i}$ the measured data of motion-set $i$. It is well-known that the optimization problem is illposed and does need some regularization. Here, we have chosen as regularizer $\mathrm{R}$ the TV functional, which is widely used in image processing [11]. Furthermore, the image $\mathbf{f}$ has only non-negative values, which is explicitly formulated in the constraint. To find a minimum of equation (2), we first resolve the constrain $\mathbf{f} \geq 0$, following [12], by the substitution $\mathbf{f}=e^{\mathbf{z}}$. Afterwards we use a Newton method [13] to minimise iterative equation (2) by solving

$$
\nabla^{2} J\left(\begin{array}{l}
\mathbf{z} \\
\gamma
\end{array}\right)\left(\begin{array}{l}
\mathbf{z}_{u} \\
\gamma_{u}
\end{array}\right)=-\nabla J\left(\begin{array}{l}
\mathbf{z} \\
\gamma
\end{array}\right)
$$

in every iteration to update the actual solution $(\mathbf{z}, \gamma)^{T}$ with $\left(\mathbf{z}_{u}, \gamma_{u}\right)^{T}$

\section{Results}

To clarify the power of our new approach we are choosing in a first step $\gamma$ as a rigid motion model and compare it to the DDMC approach. Therefor three 2D academic examples were created (Fig. 1). Every image is of size $64 \times 64$ pixels and perturbed with noise. For every image we simulate a SPECT imaging with 60 projections and two abrupt rigid movements, one after projection 20 , the other after projection 40. Due to the fact that the DDMC approach need to know when motion occur we also use this information in our approach, so we can set $K=3$ in equation (2). Fig. 1 presents the results for all three tests. It shows that the results of our new approach are comparable to the quality of DDMC. To demonstrate that our approach is more powerful than DDMC we are calculating the same tests with $K=$ \#projections $=60$. This means we use no information after which projection motion occurs. The DDMC approach can not be started without this information but our approach handle this situation (Fig. 2) only with the restriction that the method has no information about the original position of the object, resulting in a rotated or shifted position. Additionally we present a test for gradual motion in Fig. 3, simulating a SPECT imaging with a translation of the object after every projection. So each projection belongs to a different object position. Due to this the DDMC approach has not enough information for the needed partial reconstruction and can not calculate a motion corrected reconstruction. From the presented results one can see that 


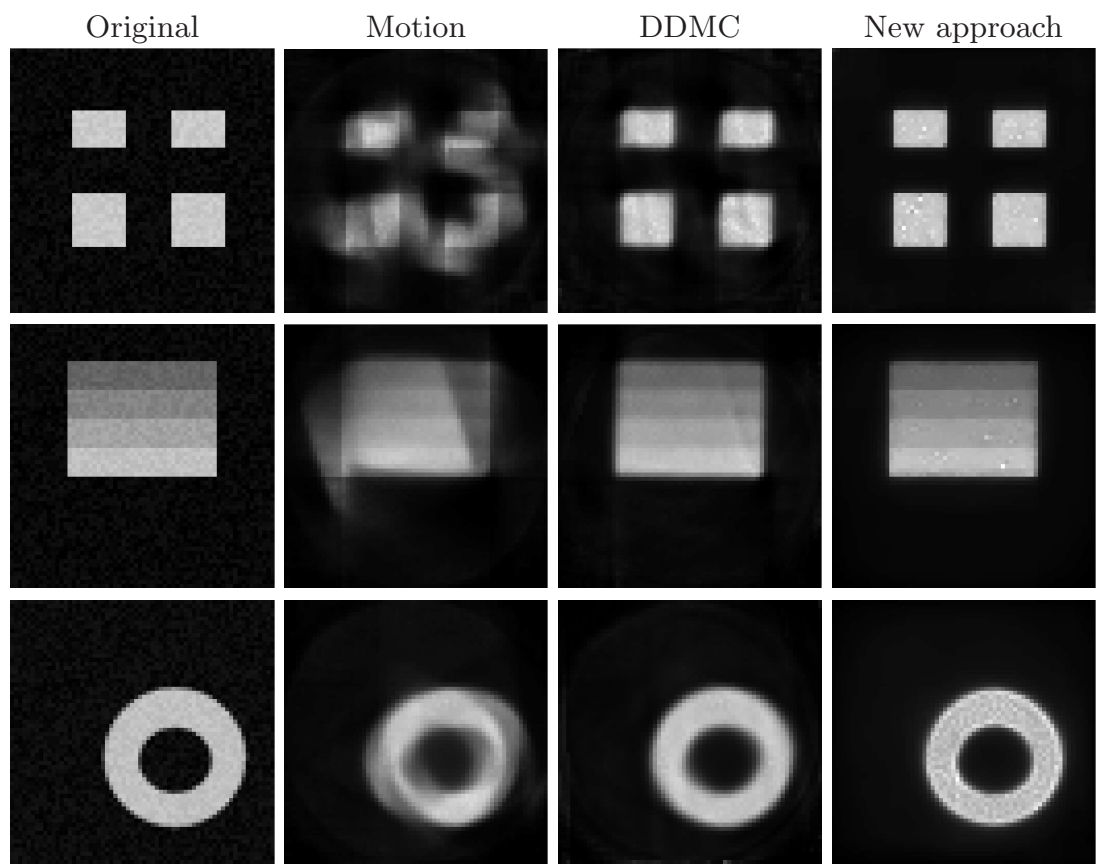

Fig. 1. The SPECT imaging of three academic examples (Original) are perturbed with abrupt rigid motion and reconstructed (Motion). Using the DDMC or the new approach the motion can be corrected
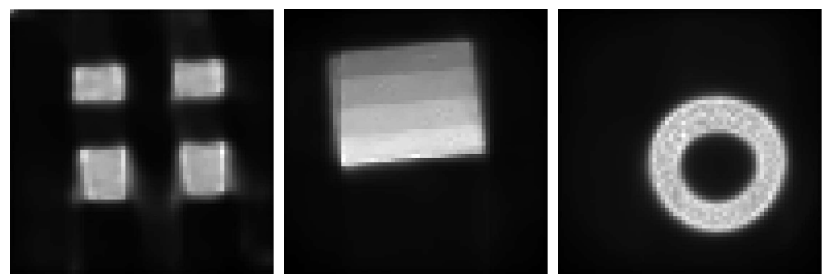

Fig. 2. Motion correction with the new approach of the examples known from Fig. 1 with $K=60$
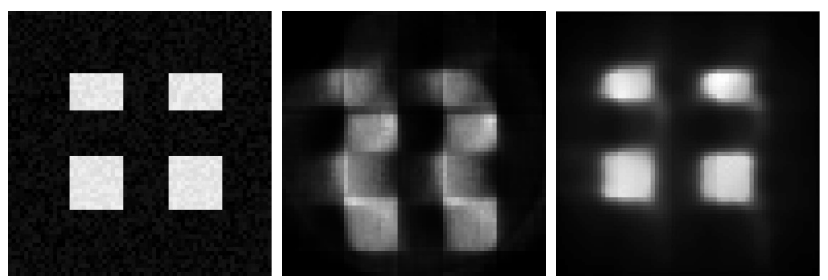

Fig. 3. A SPECT imaging of an academic example (left) is perturbed with gradual motion and reconstructed (middle). Using the new approach this motion can be corrected (right) 
our approach can not only correct abrupt motion without any information after which projection motion occurs, it can also correct gradual motion. Due to this it overcomes all restrictions of all other motion correction approaches working only with the raw data of a SPECT imaging.

\section{Discussion}

We presented a new approach for motion correction in SPECT imaging, which can correct abrupt and gradual rigid object motion. The power of this approach was clarified with some academic $2 \mathrm{D}$ examples. Our next step will be a $3 \mathrm{D}$ implementation and further tests with academic examples. Afterwards tests with real patient data are planed.

\section{References}

1. Wheat JM, Currie GM. Incidence and characterization of patient motion in myocardial perfusion SPECT: Part 1. J Nucl Med Technol 2004;32(2):60-65.

2. Botvinick EH, Zhu YY, O'Connell WJ, Dae MW. A quantitative assessment of patient motion and its effect on myocardial perfusion SPECT images. J Nucl Med 1993;34(2):303-310.

3. Cooper JA, Neumann PH, McCandless BK. Effect of patient motion on tomographic myocardial perfusion imaging. J Nucl Med 1992;33(8):1566-1571.

4. Friedman J, van Train K, Maddahi J, Rozanski A, Prigent F, Bietendorf J, et al. "Upward creep" of the heart: A frequent source of false-positive reversible defects during thallium-201 stress-redistribution SPECT. J Nucl Med 1989;30(10):17181722 .

5. Pellot-Barakat C, Ivanovic M, Weber DA, Herment A, Shelton DK. Motion detection in triple scan SPECT imaging. IEEE Trans Nucl Sci 1998;45(4):2238-2244.

6. Passalaqua AM, Narayanaswamy R. Patient motion correction of SPECT images: dual scan approach. IEEE Proc NSSS'94, Norfolk, VA 1995;3:1270-1274.

7. Lee KJ, Barber DC. Use of forward projection to correct patient motion during SPECT imaging. Phys Med Biol 1998;43:171-187.

8. Chen QS, Franken PR, Defrise M, Jonckheer MH, Deconinck F. Detection and correction of patient motion in SPECT imaging. J Nucl Med Technol 1993;21(4):198205.

9. Fulton RR, Eberl S, Meikle SR, Hutton BF, Braun M. A practical 3D tomographic method for correcting patient head motion in clinical SPECT. IEEE Trans Nucl Sci 1999;46(3):667-672.

10. Kyme AZ, Hutton BF, Hatton RL, Skerrett DW, Barnden LR. Practical aspects of a data-driven motion correction approach for brain SPECT. IEEE Trans Med Imag 2003;22(6):722-729.

11. Vogel CR, Oman ME. Fast numerical methods for total variation minimization in image reconstruction. Procs SPIE 1995.

12. Hanke M, Nagy JG, Vogel C. Quasi-Newton approach to nonnegative image restoration. Linear Algebra and its Applications 2000;316:223-236.

13. Nocedal J, Wright SJ. Numerical Optimization. Springer; 1999. 